\title{
Effects of a diet with polyphenol-rich cereal supplementation on the function and redox state of peritoneal leucocytes from mice: differences between a short ( 5 weeks) and long ( 20 weeks) period of supplementation
}

\author{
P. Álvarez, C. Alvarado, L. Jimenez and M. de la Fuente \\ Department of Animal Physiology, Faculty of Biology, Complutense University of Madrid, 28040 Madrid, Spain
}

\begin{abstract}
Although intake of natural products is a matter of great importance in relation to health, little attention is focused on the key role of cereal polyphenols in this respect. It has been shown that many of these compounds, especially as a mixture, exert a high antioxidant activity and synergistic effects. However, the protective role of a short-term and long-term intake of polyphenol-rich cereals on immune function and cellular redox state is still poorly understood. Thus, the aim was to investigate the effects of 5 and 20 weeks of supplementation $(200 \mathrm{~g} / \mathrm{kg})$ with four different cereal fractions on a population of female CD1 mice. Control animals received the standard diet without supplementation. Several indices of the function and redox state of peritoneal leucocytes were evaluated. The cereals, designated B (wheat germ), C (buckwheat flour), D (fine rice bran) and E (wheat middlings), contained a complex mixture of gallic acid, p-hydroxybenzoic acid, vanillic acid, sinapic acid, p-coumaric acid, ferulic acid, quercetin, catechin, rutin and oryzanol as the major polyphenolic compounds. In general, the polyphenol-rich cereals improved the immune function (by increasing chemotaxis capacity, lymphoproliferative response to mitogens and IL-2 levels) and the redox state (by increasing catalase antioxidant activity and decreasing oxidised glutathione:reduced glutathione, TNF $\alpha$ and malondialdehyde levels) of peritoneal leucocytes from mice. The control animals from the 20 weeks supplementation study (middle-age) showed typical age-related changes in their leucocyte functions and redox state. In several cases the antioxidant supplement administration for 20 weeks to middle-aged mice restored values to those of the adult controls. Similar effects of the cereal fractions were observed, but these effects were quantitatively more marked following long-term ( 20 weeks) compared with short-term (5 weeks) supplementation.
\end{abstract}

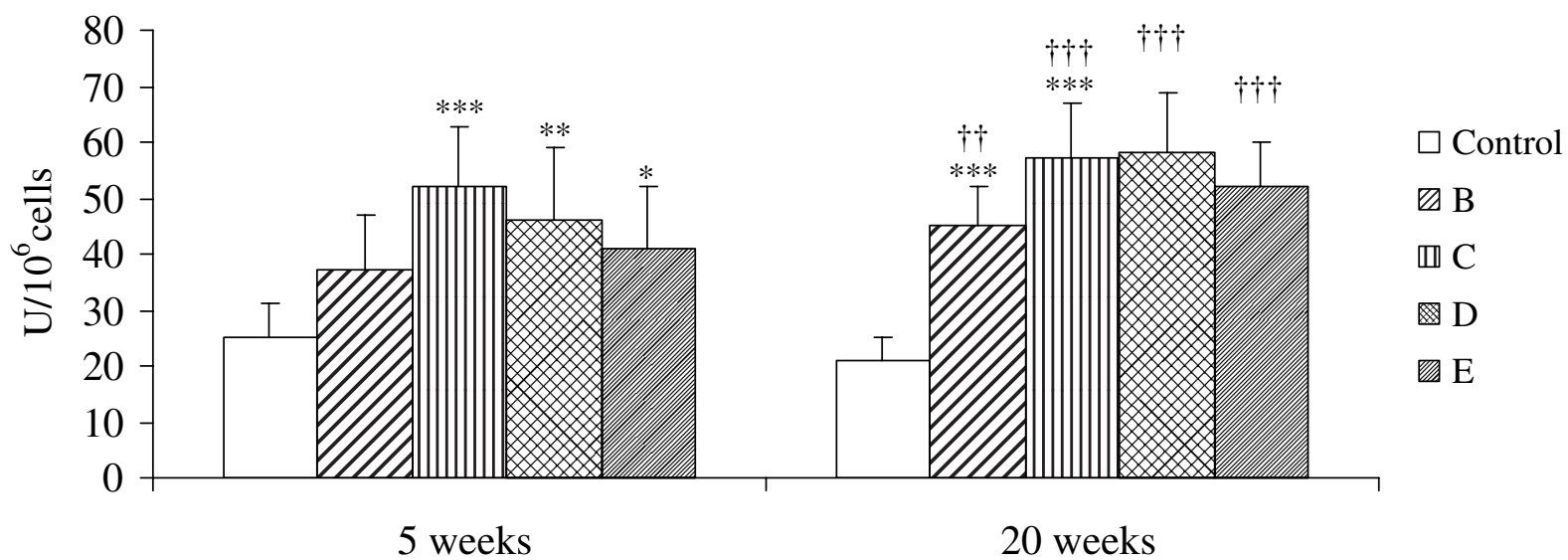

Figure. Catalase activity in peritoneal leucocytes from mice supplemented for 5 weeks and 20 weeks with polyphenol-rich cereal fractions (B, C, D or E). Values are means and standard deviations represented by vertical bars for eight ( 5 weeks) and seven (20 weeks) animals. Mean values were significantly different from those for the corresponding control group: $* P<0.05, * * P<0.01, * * * P<0.001$. Mean values were significantly different from those for the adult control group: $\dagger \dagger P<0.01$, $\dagger \dagger \dagger P<0.001$.

In conclusion, the present results suggest that polyphenol-rich cereal supplementation, especially designed for long-term intake, may be an important tool for optimizing the immune function and the redox state of leucocytes. Further studies are needed to determine the optimal doses and to establish which are the key compounds involved in mediating the observed beneficial effects before their regular consumption can be recommended.

This work was supported by MEC (BFU 2005-06777), RETICEF (RD06/0013/0003) (ISCIII) of Spain and DANONE VITAPOLE (France). 